PROCEEDINGS OF THE

AMERICAN MATHEMATICAL SOCIETY

Volume 140, Number 10, October 2012, Pages 3397-3408

S 0002-9939(2012)11251-1

Article electronically published on February 23, 2012

\title{
ON SOME MODULI SPACES OF BUNDLES ON $K 3$ SURFACES, II
}

\author{
C. G. MADONNA
}

(Communicated by Lev Borisov)

\begin{abstract}
We give several examples of the existence of infinitely many divisorial conditions on the moduli space of polarized $K 3$ surfaces $(S, H)$ of degree $H^{2}=2 g-2, g \geq 3$, and Picard number $\rho(S)=r k N(S)=2$, such that for a general $K 3$ surface $S$ satisfying these conditions the moduli space of sheaves $M_{S}(r, H, s)$ is birationally equivalent to the Hilbert scheme $S[g-r s]$ of zerodimensional subschemes of $S$ of length equal to $g-r s$. This result generalizes a result of Nikulin when $g=r s+1$ and an earlier result of the author when $r=s=2, g \geq 5$.
\end{abstract}

\section{INTRODUCTION TO THE MAIN RESULT}

Let $S \subset \mathbf{P}^{g}$ be a smooth projective $K 3$ surface of genus $g \geq 3$ and $H$ be a general hyperplane section of $S, H^{2}=2 g-2$. Let us denote by $\widetilde{H}(S, \mathbf{Z})$ the Mukai lattice of $S$ and let $v=\left(v_{0}, v_{1}, v_{2}\right) \in \widetilde{H}(S, \mathbf{Z})$ be an algebraic primitive Mukai vector (i.e. $\mathbf{Z} v$ is a primitive sublattice of $\widetilde{H}(S, \mathbf{Z})$ and $v_{1} \in N(S)$, the Picard lattice of $\left.S\right)$. We briefly recall that the Mukai lattice of $S$ is the full cohomology group

$$
\widetilde{H}(S, \mathbf{Z}):=H^{0}(S, \mathbf{Z}) \oplus H^{2}(S, \mathbf{Z}) \oplus H^{4}(S, \mathbf{Z})
$$

endowed by the Mukai pairing

$$
(v, w)=v_{1} \cdot w_{1}-\left(v_{0} w_{2}+v_{2} w_{0}\right) \in \mathbf{Z},
$$

where $v=\left(v_{0}, v_{1}, v_{2}\right), w=\left(w_{0}, w_{1}, w_{2}\right) \in \widetilde{H}(S, \mathbf{Z}), H^{0}(S, \mathbf{Z})$ and $H^{4}(S, \mathbf{Z})$ are naturally identified with $\mathbf{Z}$, and the transcendental lattice of $S$ is defined by $T(S)=$ $N(S)^{\perp} \subset H^{2}(S, \mathbf{Z})$; i.e., it is the orthogonal lattice to the Picard lattice $N(S)$ with respect to the intersection product of $H^{2}(S, \mathbf{Z})$.

We extensively studied the problem of finding conditions on $S$ such that the moduli space $M_{S}(v)$ of $H$-stable sheaves over $S$ with Mukai vector $v$ is isomorphic to the $K 3$ surface $S$ when $v$ is isotropic, i.e. $(v, v):=v^{2}=0$, and primitive. The interested reader can refer to [4] and the references given there for more details. In 2] we started to consider the case of primitive and not necessarly isotropic Mukai vectors. Indeed, by the work of Yoshioka and Mukai (see [12, [5]), the moduli space $M_{S}(v)$ is an irreducible symplectic variety of dimension equal to $v^{2}+2$ which is

Received by the editors August 17, 2010 and, in revised form, April 12, 2011.

2010 Mathematics Subject Classification. Primary 14D20, 14J28.

The author was supported by EPSRC grant EP/D061997/1. The author is a member of project MTM2007-67623, founded by the Spanish MEC.

(C)2012 American Mathematical Society Reverts to public domain 28 years from publication 
equivalent under deformations to the Hilbert scheme $S\left[v^{2} / 2+1\right]$ of zero-dimensional subschemes $Z$ of $S$ of length $l(Z)=v^{2} / 2+1$. The question we want to answer here is the following:

Question 1.1. Let $v \in \widetilde{H}(S, \mathbf{Z})$ be an algebraic and primitive Mukai vector and let $M:=M_{S}(v)$ be the moduli space of $H$-stable sheaves over $S$ with Mukai vector $v$. Let $M^{\prime}:=S\left[v^{2} / 2+1\right]$ be the Hilbert scheme of zero-dimensional subschemes of $S$ of length $v^{2} / 2+1$. When is $M$ birational to $M^{\prime}$ ?

In this paper we will refer to a birational map between $M$ and $M^{\prime}$ as a "birational isomorphism" or just "isomorphism" for short. We are mainly interested here in the case when $v^{2}>0$. When $v^{2}=0$, a complete answer was given in [4, 8] and 9] for a general $K 3$ surface $S$ of Picard number $\rho(S):=\operatorname{rk} N(S) \leq 2$ (i.e. with $\left.\operatorname{Aut}\left(T(S), H^{2,0}(S)\right)= \pm 1\right)$.

For any $g \geq 5$ we have already considered the previous question in 2 for the case of the Mukai vector $(2, H, 2)$. We found there infinitely many divisorial conditions on the moduli space of polarized $K 3$ surfaces of degree $H^{2}=2 g-2$ for which one has a birational isomorphism $M \cong M^{\prime}$. Here we partially answer the previous question when the Mukai vector is $v=(r, H, s)$ and the following conditions are satisfied:

$$
\left\{\begin{array}{l}
r, s \in \mathbf{Z}, r, s \geq 1 \\
H^{2}=2 g-2 \\
\rho(S)=2 \\
\gamma(H)=1 \\
\text { either } r \mid 2 \text { or } r \mid g-1, \text { or } s \mid g-1 \text { or } s \mid 2,
\end{array}\right.
$$

where $\gamma(H) \in \mathbf{N}$ is defined by $H \cdot N(S) \cong \gamma(H) \mathbf{Z}$.

Since $H^{2}=2 g-2$ we have $(v, v)=2 g-2-2 r s=2(g-1-r s)$ and the moduli space of $H$-stable sheaves $E$ with Mukai vector $v(E)=v=(r, H, s)$,

$$
M=M_{S}(v)
$$

has dimension equal to $2(g-r s)$. The corresponding Hilbert scheme is given by

$$
M^{\prime}=S[g-r s]
$$

We recall two beautiful examples given by Mukai in which for general $S$ (say $N(S) \cong$ $\mathbf{Z}[H]$ ) one cannot hope to get any isomorphism between $M$ and $M^{\prime}$. The first one corresponds to the case $g=5, r=s=2$. In this case $v$ is isotropic and the surface $S=M^{\prime}$ is an octic $K 3$ complete intersection of three quadrics in $\mathbf{P}^{5}$ while $M$ is a $K 3$ double plane, i.e. a ramified sextic double plane, isomorphic to the double covering of the net of quadrics whose base locus defines $S$ ramified over the locus of singular quadrics of the net (see 3 for more detials on this case). Of course in general $M^{\prime}=S \neq M=M_{S}(2, H, 2)$ since $\operatorname{det} N(M)=2 \neq \operatorname{det} N(S)=8$.

The second example, also related to the geometry of quadrics, is a 4-dimensional analogue to the above one, corresponding to the case $g=6, r=s=2$. The interested reader can refer to [6] for more details.

In this short paper we give several examples of infinitely many divisorial conditions on the moduli space of polarized $K 3$ surfaces $(S, H)$ of degree $H^{2}=2 g-2$ 
for which one gets a birational isomorphism between $M$ and $M^{\prime}$. The main tool will be considering the following isometries of the Mukai lattice of $S$ : by

$$
T_{D}\left(r, c_{1}, s\right)=\left(r, c_{1}+r D, s+r\left(D^{2} / 2\right)+D \cdot c_{1}\right)
$$

and

- the reflection $\delta: \widetilde{H}(S, \mathbf{Z}) \rightarrow \widetilde{H}(S, \mathbf{Z})$ defined by

$$
\delta\left(r, c_{1}, s\right)=\left(s, c_{1}, r\right) \text {. }
$$

Indeed when $c_{1}=H$, if $w:=T_{D}(r, H, s)$, then one gets an isomorphism $M_{S}(r, H, s)$ $\cong M_{S}(w)$. One also has an isomorphism $M_{S}(r, H, s) \cong M_{S}(s, H, r)$.

Further we will mainly need to distinguish two cases. For this we introduce the following general definition.

Definition 1.1. Let $v=(r, H, s) \in \widetilde{H}(S, \mathbf{Z})$. We say that $v$ is of type + if there exists a divisor $D \in N(S)$ such that $v^{+}:=T_{D}(v)=(r, H+r D, 1)$. We say that $v$ is of type - if there exists a divisor $D \in N(S)$ such that $v^{-}:=T_{D}(v)=$ $(r, H+r D,-1)$.

Since $M_{S}(v) \cong M_{S}\left(v^{ \pm}\right)$, in order to answer our main question we will answer the following:

Question 1.2. Under which conditions on the Picard lattice $N(S)$ of the polarized $K 3$ surface $S$ of degree $H^{2}=2 g-2$ is the Mukai vector $v=(r, H, s)$ of type \pm and $M_{S}\left(v^{ \pm}\right) \cong S[g-r s] ?$

If $v$ is of type + , then applying the reflection $\delta$, we get that

$$
M_{S}(v) \cong M_{S}(r, H+r D, 1)=M_{S}\left(v^{+}\right) \cong M_{S}(1, H+r D, r) \cong S[g-r s] .
$$

Hence, in this case we are done if we are able to find the divisor $D$ as above such that $T_{D}(r, H, s)=(r, H+r D, 1)$. When $v$ is of type - we construct explicitly the required birational isomorphism $M_{S}\left(v^{-}\right) \cong S[g-r s]$.

As in 8 , given a $K 3$ surface $S$, we introduce the invariant $\pm \mu \bmod (2 g-2)$ of the pair $(H, N)$, where $N=N(S)$ is the Picard lattice of $S$ and $H \in N$, $H^{2}=2 g-2, \gamma(H)=1$. If $\mathbf{Z} G$ is the orthogonal complement to $H$ in $N$, then $(\mu H+G) /(2 g-2) \in N$ for some $\mu \in \mathbf{Z}$ (since $\gamma(H)=1)$. If $-d=\operatorname{det}(N)$, then $\mu^{2} \equiv d \bmod (4 g-4)$, and $\pm \mu \bmod (2 g-2) \in(\mathbf{Z} /(2 g-2))^{*}$. Thus the pair $(H, N)$ of the $K 3$ surface $S$ has the invariants $(g, d, \pm \mu \bmod (2 g-2)$ ) (see Section 2 for more details).

Our first result is the following:

Proposition 1.1. Let $S$ be a polarized $K 3$ surface of degree $H^{2}=2 g-2$, general with $\rho(S)=2, \gamma(H)=1, \operatorname{det} N(S)=-d, d \equiv \mu^{2} \bmod 4(g-1), \pm \mu \bmod 2 g-2 \in$ $(\mathbf{Z} /(2 g-2))^{*}$. Let $v=(r, H, s)$ and let

$$
\begin{gathered}
\mathcal{D}_{ \pm}=\left\{d \in \mathbf{N}: d=\frac{(r x+2(g-1))^{2}-4(g-1)( \pm r-r s+g-1)}{r^{2} y^{2}},\right. \\
x, y \in \mathbf{Z}, x \equiv \mu y \bmod 2 g-2\} .
\end{gathered}
$$

Then if $d \in \mathcal{D}_{ \pm}$, the Mukai vector $v$ is of type $v^{ \pm}$. Indeed we have that $v^{ \pm}=$ $(r, F, \pm 1)$, where

$$
F:=H+r D
$$


with $F \cdot H \equiv r \mu y \bmod 2 g-2$ and $D=(x H+y G) /(2 g-2) \in N(S),(r x+$ $2(g-1))^{2}-d r^{2} y^{2}=4(g-1)( \pm r-r s+g-1)$. Moreover if either $r \mid g-1$ or $r \mid 2$, then $\mathcal{D}_{ \pm}$is infinite containing the infinite subset

$$
\begin{aligned}
\mathcal{D}_{ \pm}^{1}=\{d & \in \mathbf{N}: d=\frac{(r x+2(g-1))^{2}-4(g-1)( \pm r-r s+g-1)}{r^{2}}, \\
x & \in \mathbf{Z}, x \equiv \mu \bmod 2 g-2\},
\end{aligned}
$$

and there exist infinitely many $d \in \mathcal{D}_{ \pm}^{1}$ such that the corresponding divisor $D=$ $(x H+G) /(2 g-2)$ satisfies the condition: $D \cdot H \ll 0$.

Using the above result we then derive the main result of the paper:

Theorem 1.1. Let $S$ be a general K3 surface of degree $H^{2}=2 g-2$ with Picard lattice $N(S):=N$ and Picard number $\rho(S)=2, \gamma(H)=1$, $\operatorname{det} N=-d, d \equiv \mu^{2}$ $\bmod 4(g-1)$ and $\pm \mu \bmod 2 g-2 \in(\mathbf{Z} /(2 g-2))^{*}$. If $r \mid 2$ or $r \mid g-1$, then there exist infinitely many $d \in \mathcal{D}_{ \pm}^{1}$ such that

(1) there exists a divisor $D=(x H+G) /(2 g-2) \in N$ as in the previous proposition such that the divisor $F=H+r D \in N(S)$ satisfies

$$
F^{2}=(2 g-2)+r( \pm 2-2 s)
$$

and $D \cdot H \ll 0, F \cdot H \equiv r \mu \bmod 2 g-2,(r x+2(g-1))^{2}-d r^{2}=$ $4(g-1)( \pm r-r s+g-1)$

(2) there exists a birational isomorphism $M_{S}(r, H, s) \cong S[g-r s]$.

From the proof of the theorem it follows that the above birational isomorphism is obtained by the composition of the isomorphism

$$
\varphi(D)_{ \pm}: M_{S}(r, H, s) \rightarrow M_{S}(r, H+r D, \pm 1)
$$

induced by the tensorization for the line bundle $D$ as in (1) and:

(a) when $d \in \mathcal{D}_{+}^{1}$, the isomorphism $\delta: M_{S}(r, H+r D, 1) \rightarrow M_{S}(1, H+r D, r)$ induced by the reflection $\delta$ (see (1.7)), and the isomorphism $\varphi_{+}: M_{S}(1, H+r D, r) \rightarrow$ $S[g-r s]$ inverse of the isomorphism $\varphi_{+}^{-1}: S[g-r s] \rightarrow M_{S}(1, H+r D, r)$ defined by $\varphi_{+}^{-1}(Z) \cong \mathfrak{I}_{Z}(F)$; actually this isomorphism exists for all $d \in \mathcal{D}_{+}^{1}$;

(b) when $d \in \mathcal{D}_{-}^{1}$, with a birational isomorphism $\varphi_{-}: M_{S}(r, H+r D,-1) \cong$ $S[g-r s]$ inverse of a birational isomorphism $\left(\varphi_{-}\right)^{-1}: S[g-r s] \cong M_{S}(r, H+r D,-1)$ which is explicitly given in the proof of Theorem 1.1 .

It is important to notice that under the conditions of the previous theorem one can apply the reflection $\delta$ to get a birational isomorphism $M_{S}(r, H, s) \cong$ $M_{S}(s, H, r)$. Then, arguing in a similar way, one can show the following: if $s \mid g-1$ or $s \mid 2$, then there exist infinitely many $d \in \widetilde{\mathcal{D}}_{ \pm}^{1}$, where

$$
\begin{gathered}
\widetilde{\mathcal{D}}_{ \pm}^{1}=\left\{d \in \mathbf{N}: d=\frac{(s x+2(g-1))^{2}-4(g-1)( \pm s-r s+g-1)}{s^{2}},\right. \\
\qquad \widetilde{\mathcal{D}}_{ \pm}=\{d \in \mathbf{Z}, x \equiv \mu \bmod 2 g-2\} \\
s^{2} y^{2} d=\frac{(s x+2(g-1))^{2}-4(g-1)( \pm s-r s+g-1)}{x \in \mathbf{Z}, x \equiv \mu y \bmod 2 g-2\}}
\end{gathered}
$$

such that $M_{S}(r, H, s) \cong S[g-r s]$. In particular for all such $d$ there exists a divisor $\widetilde{D}=(x H+G) /(2 g-2) \in N(S)$ such that the divisor

$$
\widetilde{F}=H+s \widetilde{D}
$$


satisfies

$$
\widetilde{F}^{2}=(2 g-2)+s( \pm 2-2 r)
$$

and $\widetilde{D} \cdot H \ll 0, \widetilde{F} \cdot H \equiv s \mu \bmod 2 g-2,(s x+2(g-1))^{2}-d s^{2}=4(g-1)( \pm s-r s+g-1)$. We leave it to the reader to write a statement similar to the one of our main theorem in this case.

We remark that if $r=s$, then $\mathcal{D}_{ \pm}=\widetilde{\mathcal{D}}_{ \pm}$and also $\mathcal{D}_{ \pm}^{1}=\widetilde{\mathcal{D}}_{ \pm}^{1}$

Remember also when $H^{2}=2 g-2=2 r s$, i.e. the Mukai vector $v=(r, H, s)$ is isotropic and the moduli space $M_{S}(v)$ is a $K 3$ surface, we have that the corresponding divisor $F$ satisfies the conditions

$$
F^{2}= \pm 2 r, F \cdot H \equiv 0 \quad \bmod r
$$

and the divisor $\widetilde{F}$ satisfies the conditions

$$
\widetilde{F}^{2}= \pm 2 s, \widetilde{F} \cdot H \equiv 0 \bmod s .
$$

In this case in 8 , using the Global Torelli theorem for $K 3$ surfaces, it was proved that these two conditions (1.16) and (1.17) are not only sufficient but also necessary to have the isomorphism $S \cong M_{S}(v)$ when $S$ is general with $\rho(S) \leq 2$. Our result here in the isotropic case is weaker with respect to the one obtained in $[8$; nevertheless it generalizes to the nonisotropic case the known results obtained for the case of isotropic Mukai vectors.

Our main result also gives the following interesting:

Corollary 1.1. Under the conditions and notation of Theorem 1.1 , let $\mathcal{D}=\mathcal{D}_{+} \cup$ $\mathcal{D}_{-}$and $\widetilde{\mathcal{D}}=\widetilde{\mathcal{D}}_{+} \cup \widetilde{\mathcal{D}}_{-}$. If $r \mid g-1$ or $s \mid g-1$ or $r \mid 2$ or $s \mid 2$, then $\mathcal{D} \cup \widetilde{\mathcal{D}}$ is infinite containing the infinite subset $\mathcal{D}^{1} \cup \widetilde{\mathcal{D}}^{1}$, where $\mathcal{D}^{1}=\mathcal{D}_{+}^{1} \cup \mathcal{D}_{-}^{1}, \widetilde{\mathcal{D}}^{1}=\widetilde{\mathcal{D}}_{+}^{1} \cup \widetilde{\mathcal{D}}_{-}^{1}$.

Note that if $g-1=r s$, i.e. $v=(r, H, s)$ is isotropic, it then follows that $\mathcal{D}$ and $\widetilde{\mathcal{D}}$ are infinite. This is also the case when $g \geq 5$ and $v=(2, H, 2)$ (see [2]).

It is interesting to rewrite our previous result in terms of the Beauville-Bogomolov quadratic form $q$ (and bilinear form $b$ ) on $H^{2}(S[g-r s], \mathbf{Z})$ (see e.g. 1 for details). Remember that $H^{2}(S[g-r s], \mathbf{Z}) \cong H^{2}(S, \mathbf{Z}) \oplus_{\perp} \mathbf{Z} f$, with $f^{2}=-2(g-r s-1)$. The corollary below shows how our main result here can be considered as a natural generalization to nonisotropic Mukai vectors of the result given in 8 for the case of isotropic Mukai vectors.

Corollary 1.2. Under the conditions and notation of Theorem 1.1 there exist infinitely many $d \in \widetilde{\mathcal{D}} \cup \mathcal{D}$, such that there exists either a divisor class $F \in N(S)$ as in (1.10) such that

$$
h_{1}=F+\epsilon f \in H^{2}(S[g-r s], \mathbf{Z})
$$

satisfies

$$
q\left(h_{1}\right)= \pm 2 r, b\left(h_{1}, H\right)=F \cdot H \equiv r \mu \quad \bmod 2 g-2, b\left(h_{1}, H\right) \ll 0
$$

when $(r x+2(g-1))^{2}-d r^{2}=4(g-1)( \pm r-r s+g-1)$ or a divisor class $\widetilde{F}$ as in (1.14) such that the element

$$
\widetilde{h}_{1}=\widetilde{F}+\epsilon f \in H^{2}(S[g-r s], \mathbf{Z})
$$

satisfies

$$
q\left(\widetilde{h}_{1}\right)= \pm 2 s, b\left(\widetilde{h}_{1}, H\right)=\widetilde{F} \cdot H \equiv s \mu \quad \bmod 2 g-2, b\left(\widetilde{h}_{1}, H\right) \ll 0
$$


when $(s x+2(g-1))^{2}-d s^{2}=4(g-1)( \pm s-r s+g-1)$. Here $\epsilon=0$ when $g=r s+1$ and $\epsilon=1$ when $g>r s+1$.

\section{A PROOF OF THE MAIN RESUlT}

In the previous section we stated our main result. We also made several remarks, derived some corollaries, and posed some questions we hope to answer in further works. It remains now to give a proof of Theorem 1.1. In this section we will do this. We will now give the proof of Proposition 1.1, which will be needed for the proof of Theorem 1.1 .

At first, to fix notation and terminology, we will recall some basic facts on lattices (see 7 for more details) we will need in the sequel. A lattice $L$ is a nondegenerate integral symmetric bilinear form; i.e. $L$ is a free $\mathbf{Z}$-module equipped with a symmetric pairing $x \cdot y \in \mathbf{Z}$ for $x, y \in L$, and this pairing should be non-degenerate. We denote $x^{2}=x \cdot x$. The determinant of $L$ is defined to be $\operatorname{det} L=\operatorname{det}\left(e_{i} \cdot e_{j}\right)$, where $\left\{e_{i}\right\}$ is some basis of $L$. We will use the notation $\left[x_{1}, \ldots, x_{n}\right]$ to mean the lattice generated by the elements $x_{1}, \ldots, x_{n}$.

An isometry of lattices is an isomorphism of modules which preserves the pairing.

As in 8 , given a $K 3$ surface $S$, we introduce the invariant $\pm \mu \bmod (2 g-2)$ of the pair $(H, N)$ where $N=N(S)$ is the Picard lattice of $S$ and $H \in N, H^{2}=$ $2 g-2, \gamma(H)=1$, where $\gamma(H) \in \mathbf{N}$ is defined by $H \cdot N(S) \cong \gamma(H) \mathbf{Z}$. If $\mathbf{Z} G$ is the orthogonal complement to $H$ in $N$, then $(\mu H+G) /(2 g-2) \in N$ for some $\mu \in \mathbf{Z}$ (since $\gamma(H)=1)$. If $-d=\operatorname{det}(N)$, then $\mu^{2} \equiv d \bmod (4 g-4)$, and $\pm \mu$ $\bmod (2 g-2) \in(\mathbf{Z} /(2 g-2))^{*}$. Thus the pair $(H, N)$ of the $K 3$ surface $S$ has the invariants $(g, d, \pm \mu \bmod (2 g-2))$.

For the rest of this section we will assume that the following conditions on the Picard lattice $N(S)$ of $S$ are satisfied:

$$
\left\{\begin{array}{l}
\gamma(H)=1 \\
\rho(S)=2, \\
\pm \mu \bmod 2 g-2 \in(\mathbf{Z} /(2 g-2))^{*}
\end{array}\right.
$$

We also need the following (see 8 for a proof):

Proposition 2.1. Let $S$ be a $K 3$ surface with a polarization $H$ of degree $H^{2}=$ $2 g-2$. Assume that conditions (2.1) hold. Then the lattice $N(S)$ is defined by its determinant $\operatorname{det} N(S)=-d$, where $d \in \mathbf{N}$, and $\mu^{2} \equiv d \bmod 4(g-1)$. There exists a unique choice of a primitive vector $G$ orthogonal to $H$ such that

$$
N(S)=[H, G,(\mu H+G) /(2 g-2)],
$$

where $G^{2}=-(2 g-2) d$, and $\pm \mu \bmod 2 g-2 \in(\mathbf{Z} /(2 g-2))^{*}$. It follows that

$$
N(S)=\{(x H+y G) /(2 g-2): x, y \in \mathbf{Z}, x \equiv \mu y \quad \bmod 2 g-2\} .
$$

We can now derive the following:

Proposition 2.2. Let $S$ be a $K 3$ surface with a polarization $H$ of degree $H^{2}=$ $2 g-2$. Suppose that conditions (2.1) hold. Let $d \in \mathcal{D}_{ \pm}$, where

$$
\begin{aligned}
\mathcal{D}_{-}=\left\{d \in \mathbf{N}: d=\frac{(r x+2(g-1))^{2}-4(g-1)(-r-r s+g-1)}{r^{2} y^{2}},\right. \\
x, y \in \mathbf{Z}, x \equiv \mu y \quad \bmod 2 g-2\},
\end{aligned}
$$




$$
\begin{gathered}
\mathcal{D}_{+}=\left\{d \in \mathbf{N}: d=\frac{(r x+2(g-1))^{2}-4(g-1)(r-r s+g-1)}{r^{2} y^{2}},\right. \\
x, y \in \mathbf{Z}, x \equiv \mu y \bmod 2 g-2\}
\end{gathered}
$$

Then there exists a divisor $D=(x H+y G) /(2 g-2) \in N(S)$ such that $T_{D}(r, H, s)=$ $(r, H+r D, \pm 1)$ and $(r x+2(g-1))^{2}-d r^{2} y^{2}=4(g-1)( \pm r-r s+g-1)$. For all such $d$ the divisor $D$ as above is then given by $D=(x H+y G) /(2 g-2)$, and we have that $F:=H+r D$ satisfies

$$
F^{2}=(2 g-2)+r( \pm 2-2 s)
$$

with $F \cdot H \equiv r \mu y \bmod 2 g-2$. Moreover if $r \mid 2$ or $r \mid g-1$, then $\mathcal{D}_{ \pm}$is infinite containing the infinite subset

$$
\begin{aligned}
\mathcal{D}_{ \pm}^{1}=\{d & \in \mathbf{N}: d=\frac{(r x+2(g-1))^{2}-4(g-1)( \pm r-r s+g-1)}{r^{2}} \\
x & \in \mathbf{Z}, x \equiv \mu \bmod 2 g-2\}
\end{aligned}
$$

and there exist infinitely many $d \in \mathcal{D}_{ \pm}^{1}$ such that the corresponding divisor $D=$ $(x H+G) /(2 g-2)$ satisfies $D \cdot H \ll 0$.

Proof. By the previous proposition we can write a divisor $D \in N(S)$ in the following form:

$$
D=\frac{x H+y G}{2 g-2}
$$

with $x, y \in \mathbf{Z}$ and $x \equiv \mu y \bmod 2 g-2$. Then $T_{D}(r, H, s)=(r, H+r D, \pm 1)$ if and only if $(x, y)$ is an integral solution of the Pell-type equation

$$
(r x+2(g-1))^{2}-d(r y)^{2}=4(g-1)( \pm r-r s+g-1) .
$$

In particular if we denote $H+r D$ by $F$, we have that $F^{2}=(2 g-2)+r( \pm 2-2 s)$ and $F \cdot H \equiv r \mu y \bmod 2 g-2$. Finally, if $r \mid 2$ or $r \mid g-1$, we may take $d \gg 0$ and hence we may assume that $x \ll 0$, i.e. $D \cdot H \ll 0$.

We can now give a proof of our main result.

Theorem 2.1. Let $S$ be a general $K 3$ surface of degree $H^{2}=2 g-2$ with Picard lattice $N(S):=N$ and Picard number $\rho(S)=2, \gamma(H)=1$, $\operatorname{det} N=-d, d \equiv \mu^{2}$ $\bmod 4(g-1)$ and $\pm \mu \bmod 2 g-2 \in(\mathbf{Z} /(2 g-2))^{*}$. If $r \mid 2$ or $r \mid g-1$, then there exist infinitely many $d \in \mathcal{D}_{ \pm}^{1}$ such that:

(1) there exists a divisor $D=(x H+G) /(2 g-2) \in N$ as in the previous proposition such that the divisor $F=H+r D \in N(S)$ satisfies

$$
F^{2}=(2 g-2)+r( \pm 2-2 s)
$$

and $D \cdot H \ll 0, F \cdot H \equiv r \mu y \bmod 2 g-2,(r x+2(g-1))^{2}-d r^{2}=$ $4(g-1)( \pm r-r s+g-1)$;

(2) there exists a birational isomorphism $M_{S}(r, H, s) \cong S[g-r s]$.

Proof. We already noticed that if $d \in \mathcal{D}_{+}$, then the result follows soon. Indeed in this case the birational isomorphism $M^{\prime} \cong M$ is obtained as the composition $\varphi_{+} \circ \delta \circ \varphi(D)_{+}$of the isomorphism

$$
\varphi(D)_{+}: M_{S}(r, H, s) \cong M_{S}(r, H+r D, 1)
$$


given by the tensorization for the line bundles $D \in N=N(S)$ of the previous proposition, the isomorphism

$$
\delta: M_{S}(r, H+r D, 1) \cong M_{S}(1, H+r D, r)
$$

induced by the reflection (1.7), and the isomorphism

$$
\varphi_{+}: M_{S}(1, H+r D, r) \cong S[g-r s],
$$

which is the inverse of the isomorphism $\varphi_{+}^{-1}: S[g-r s] \rightarrow M_{S}(1, H+r D, r)$ defined by $\varphi_{+}^{-1}(Z)=\mathfrak{I}_{Z}(H+r D)$. This is indeed the case for all $d \in D_{+}$. Therefore we assume that $d \in \mathcal{D}_{-}$. Let $v=(r, H, s)$ be of type - and hence let $v^{-}=(r, F,-1)$, where $F=H+r D$ with $D \in N(S)$ as in the previous proposition, in particular such that $D \cdot H \ll 0$. We will give here explicitly the map $\left(\varphi_{-}\right)^{-1}: S[g-r s] \rightarrow$ $M_{S}\left(v^{-}\right)$. Then we will get the required birational isomorphism as a composition of the isomorphism

$$
\varphi(D)_{-}: M_{S}(r, H, s) \cong M_{S}(r, H+r D,-1)
$$

given by the tensorization for the line bundles $D \in N(S)$ as in the previous proposition and the birational isomorphism

$$
\varphi_{-}: M_{S}(r, H+r D,-1) \cong S[g-r s] .
$$

Let $Z \in S[g-r s]$ be a general subscheme of $S$ of length $l(Z)=g-r s$. Then $h^{1} \mathfrak{I}_{Z}(F)=r-1$. Indeed we have the short exact sequence defining $Z \subset S$,

$$
0 \rightarrow \mathfrak{I}_{Z}(F) \rightarrow \mathfrak{O}_{S}(F) \rightarrow \mathfrak{O}_{Z}(F) \rightarrow 0,
$$

and the corresponding exact sequence in cohomology. It then follows by standard calculations that $h^{1} \mathfrak{I}_{Z}(F) \geq r-1$. Following the construction of [11] we then have the exact sequence

$$
0 \rightarrow H^{1} \mathfrak{I}_{Z}(F) \otimes \mathfrak{O}_{S} \rightarrow \mathcal{E}=\mathcal{E}_{Z} \rightarrow \mathfrak{I}_{Z}(H+r D) \rightarrow 0,
$$

where $\mathcal{E}$ has $r k(\mathcal{E})=h^{1} \mathfrak{I}_{Z}(F)+1, c_{1}(\mathcal{E})=F:=H+r D$ and $\chi(\mathcal{E})=r-1$, $h^{1} \mathcal{E}=0$. Hence the Mukai vector of $\mathcal{E}$ is $v(\mathcal{E})=(r, F,-1)$. We define here $\left(\varphi_{-}\right)^{-1}(Z)=\mathcal{E}_{Z}=\mathcal{E}$. It remains to show that $\mathcal{E}$ is $H$-stable. We will show that $\mathcal{E}(-D)$ is $H$-stable. Remember that $F=H+r D$ for a suitable $D \in N(S)$ such that $D \cdot H \ll 0$. We then tensorize the above exact sequence by $\mathfrak{O}_{S}(-D)$ to get

$$
0 \rightarrow \mathfrak{O}_{S}^{r-1}(-D) \rightarrow \mathcal{E}(-D) \rightarrow \mathfrak{I}_{Z}(H+(r-1) D) \rightarrow 0 .
$$

Now let us assume that $\mathcal{E}(-D)$ is unstable. Then consider the maximal destabilizing subsheaf of $\mathcal{E}(-D)$, say $\mathcal{M}$. We have

$$
\mu(\mathcal{M})=\frac{\left(c_{1}(\mathcal{M}) \cdot H\right)}{r k(\mathcal{M})}>\mu(\mathcal{E}(-D))=\frac{2 g-2}{r}>0 .
$$

Let $i$ be the inclusion map of $\mathcal{M}$ in $\mathcal{E}(-D)$ and let $\beta: \mathcal{E}(-D) \rightarrow \mathfrak{I}_{Z}(H+(r-1) D)$ be the map given in the above exact sequence. Then the image of $\mathcal{M}$ in $\mathfrak{I}_{Z}(H+$ $(r-1) D)$ by the composite map $\beta \circ i$ is a subsheaf of rank 1 which is of type $\mathfrak{I}_{W}(H+(r-1) D)$ for some zero-dimensional subscheme $W$ containing $Z$. Then we have an exact sequence

$$
0 \rightarrow \mathcal{M}^{\prime} \rightarrow \mathcal{M} \rightarrow \mathfrak{I}_{W}(H+(r-1) D) \rightarrow 0
$$


and $c_{1}\left(\mathcal{M}^{\prime}\right)=c_{1}(\mathcal{M})-(H+(r-1) D)$. We then have

$$
\begin{aligned}
\mu\left(\mathcal{M}^{\prime}\right) & =\frac{c_{1}\left(\mathcal{M}^{\prime}\right) \cdot H}{r k\left(\mathcal{M}^{\prime}\right)}=\frac{c_{1}(\mathcal{M}) \cdot H-(H+(r-1) D) \cdot H}{r k\left(\mathcal{M}^{\prime}\right)} \\
& >\frac{c_{1}(\mathcal{M}) \cdot H}{r k\left(\mathcal{M}^{\prime}\right)} \geq \frac{c_{1}(\mathcal{M}) \cdot H}{r k(\mathcal{M})}=\mu(\mathcal{M})
\end{aligned}
$$

since $c_{1}(\mathcal{M}) \cdot H>0, D \cdot H \ll 0, \operatorname{rk}\left(\mathcal{M}^{\prime}\right)=\operatorname{rk}(\mathcal{M})-1$. Hence $\mu\left(\mathcal{M}^{\prime}\right)>\mu(\mathcal{M})$. This is impossible since we assumed $\mathcal{M}$ to be maximal destabilizing.

From the proof of the previous theorem we have the following more general statement:

Theorem 2.2. Let $S$ be a $K 3$ surface of degree $H^{2}=2 g-2$ and suppose that $N(S)$ contains a rank 2 primitive sublattice $N \subset N(S)$ with $H \in N$. Assume that $(S, N)$ satisfies the conditions of Theorem 1.1. With the same notation of Theorem 1.1] for $\mu, d, \gamma, \mathcal{D}^{1}, \widetilde{\mathcal{D}}^{1}$, if $r \mid 2$, or $s \mid 2$ or $r \mid g-1$ or $s \mid g-1$, then there exist infinitely many $d \in \mathcal{D}^{1} \cup \widetilde{\mathcal{D}}^{1}$ such that:

(1) There exists a divisor $D=(x H+G) /(2 g-2) \in N$ such that the divisor $F=H+r D$ satisfies

$$
F^{2}=(2 g-2)+r( \pm 2-2 s)
$$

and $D \cdot H \ll 0, F \cdot H \equiv r \mu \bmod 2 g-2,(r x+2(g-1))^{2}-d r^{2}=$ $4(g-1)( \pm r-r s+g-1)$ for at least one of \pm or there exists a divisor $\widetilde{D}=(x H+G) /(2 g-2) \in N$ such that the divisor $\widetilde{F}=H+s \widetilde{D}$ satisfies

$$
\widetilde{F}^{2}=(2 g-2)+s( \pm 2-2 r),
$$

and $\widetilde{D} \cdot H \ll 0, \widetilde{F} \cdot H \equiv s \mu \bmod 2 g-2,(s x+2(g-1))^{2}-d s^{2}=4(g-$ $1)( \pm s-r s+g-1)$ for at least one of \pm .

(2) There exists a birational isomorphism $M_{S}(r, H, s) \cong S[g-r s]$.

Remark 2.1. By the proof of our main theorem it follows that one gets the required isomorphism for all $d \in \mathcal{D}_{+}$. When $d \in \mathcal{D}_{-}$we got a weaker result showing the existence of infinitely many integers $d \in \mathcal{D}_{-}^{1}$, i.e. $d \gg 0$, for which one has the required isomorphism. For our proof here, the condition $d \gg 0$ is necessary if $d \in \mathcal{D}_{-}^{1}$.

Remark 2.2. When $v^{2}=0$ Nikulin gave in 9 examples of $K 3$ surfaces $S$ with Picard lattice $N(S)$ of $\operatorname{rank} \rho(S)=3$ such that $M_{S}(v) \cong S$ while $N(S)$ does not contain any rank 2 primitive sublattice $N$ as in the previous theorem, in other words, examples when $\rho=3$ in which one has an isomorphism which is not a specialization of an isomorphism between $K 3$ surfaces of Picard number 2 . These examples have all $\gamma(H) \neq 1$. We do not know if one can find similar examples when $\gamma(H)=1$ (with isotropic or nonisotropic Mukai vectors as well).

\section{EXAMPLES}

In this section we give some examples in which the condition of the main theorem $D \cdot H \ll 0$ can be weakened to derive in this way from our result also the already known results in the literature.

Let us consider the isotropic case, i.e. $v^{2}=0$ when $g=5$ and $v=(2, H, 2)$. Here $r=s=2 \mid 2$ and $r=s=2 \mid 4=g-1$, and as noticed in the introduction we have $\mathcal{D}_{ \pm}=\widetilde{\mathcal{D}}_{ \pm}$. Our main result in this case reads as follows: 
Theorem 3.1. Let $S$ be a general $K 3$ surface of degree $H^{2}=8$ with Picard lattice $N(S):=N$ and Picard number $\rho(S)=2, \gamma(H)=1$, $\operatorname{det} N=-d, d \equiv \mu^{2} \bmod 16$ and $\pm \mu \bmod 8 \in(\mathbf{Z} / 8)^{*}$, i.e. $\mu=1$ if $d \equiv 1 \bmod 16$ and $\mu=3$ if $d \equiv 9 \bmod 16$ (see Section 3.1 of $[3]$ ).

(A) There exist infinitely many $d \in \mathcal{D}_{+}^{1}$ such that:

(a) there exists a divisor $D=(x H+G) / 8 \in N$ such that the divisor $F=H+2 D$ satisfies

$$
F^{2}=-4
$$

and $D \cdot H \ll 0, F \cdot H \equiv 2 \mu y \bmod 8,(x+4)^{2}-d y^{2}=-8 ;$

(b) there exists a birational isomorphism $M_{S}(2, H, 2) \cong S$.

(B) There exist infinitely many $d \in \mathcal{D}_{-}^{1}$ such that

(a) there exists a divisor $D=(x H+G) / 8 \in N$ such that the divisor $F=H+2 D$ satisfies

$$
F^{2}=4
$$

and $D \cdot H \ll 0, F \cdot H \equiv 2 \mu y \bmod 8,(x+4)^{2}-d y^{2}=8$;

(b) there exists a birational isomorphism $M_{S}(2, H, 2) \cong S$.

In these cases we have $\mathcal{D}=\widetilde{\mathcal{D}}$, and by Corollary $1.1 \mathcal{D}$ is infinite. The two sets $\mathcal{D}_{+}$and $\mathcal{D}_{-}$are given by

$$
\mathcal{D}_{ \pm}=\left\{d \in \mathbf{N}: d=\frac{(x+4)^{2} \pm 8}{y^{2}}, x, y \in \mathbf{Z}, x \equiv \mu y \bmod 8\right\} .
$$

After changing $x+4=a$ and $y=b$, then we get the following equations:

$$
a^{2}-d b^{2}= \pm 8
$$

with $a-4 \equiv b \bmod 8$ if $d \equiv 1 \bmod 16$ and $a-4 \equiv 3 b \bmod 8$ if $d \equiv 9 \bmod 16$. When $b=1$ we then get the conditions $a \equiv 5 \bmod 8$ if $d \equiv 1 \bmod 16$ and $a \equiv 7$ $\bmod 8$ if $d \equiv 9 \bmod 16$. We then find the following infinitely many values of $d \in \mathcal{D}_{+}$of type

$$
d=a^{2}+8,
$$

i.e.

$$
\{33,57,177,233,449,537,849,969, \ldots\},
$$

which is one of the infinite sets we found in 3 . We already noticed in Remark 2.1 that one always has the isomorphism for all $d \in \mathcal{D}_{+}$, and hence in this case we can formulate the corresponding statement as in [3]. Our main result here for the case

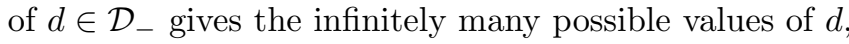

$$
\{17,41,161,217,433,449,521, \ldots\} \text {, }
$$

and is less precise than the one obtained in [3. Indeed in [3] we showed that for all $d \in \mathcal{D}_{-}$one gets the required isomorphism. Here our main result ensures the existence of an infinite subset of $\mathcal{D}_{-}$for which one gets the required isomorphism $M_{S}(2, H, 2) \cong S$. On the other hand, easy calculations (it is indeed enough to write explicitly the condition to get $D H \ll 0$ in the proof of our main theorem) show that in this case, i.e. $v=(2, H, 2)$, it is enough, for our proof given here, that the condition $D H<-8$ holds. Then, one can find explicitly that this infinite subset is equal to $D_{-} \backslash\{17,41\}$. To get the isomorphism also in these two cases $d=17,41$ one should just replace a solution $(x, 1), x>0$, with the solution $(-x-8,1)$ and consider the corresponding divisor $D$ which now satisfies the condition $D H<-8$. 
We consider now the case when $v=(2, H, 2)$ and $H^{2} \geq 10$. We have also in this case that $\mathcal{D}_{ \pm}=\widetilde{\mathcal{D}}_{ \pm}$and from our main result we derive the following statement:

Theorem 3.2. Let $S$ be a general $K 3$ surface of degree $H^{2}=2 g-2 \geq 10$ with Picard lattice $N(S):=N$ and Picard number $\rho(S)=2, \gamma(H)=1$, det $N=-d$, $d \equiv \mu^{2} \bmod 4(g-1)$ and $\pm \mu \bmod 2 g-2 \in(\mathbf{Z} /(2 g-2))^{*}$.

(A) There exist infinitely many $d \in \mathcal{D}_{+}^{1}$ such that

(a) there exists a divisor $D=(x H+G) /(2 g-2) \in N$ such that the divisor $F=H+2 D \in N$ satisfies

$$
F^{2}=2(g-7),
$$$$
F \cdot H \equiv r \mu \bmod 2 g-2,(x+g-1)^{2}-d=(g-1)(g-7) \text {; }
$$

(b) there exists a birational isomorphism $M_{S}(2, H, 2) \cong S[g-4]$.

(B) There exist infinitely many $d \in \mathcal{D}_{-}^{1}$ such that

(a) there exists a divisor $D=(x H+G) /(2 g-2) \in N$ as in the previous proposition such that the divisor $F=H+2 D \in N$ satisfies

$$
F^{2}=2(g-3)
$$

and $D \cdot H \ll 0, F \cdot H \equiv r \mu \bmod 2 g-2,(x+g-1)^{2}-d=(g-1)(g-3)$;

(b) there exists a birational isomorphism $M_{S}(2, H, 2) \cong S[g-4]$.

Using the same argument as above for the isotropic case $(2, H, 2)$, i.e. when $H^{2}=8$, one can derive the main result of 2 for any Mukai vectors $(2, H, 2)$ when $H^{2} \geq 10$ and $d \in \mathcal{D}_{+}^{1}$. Our result here extends the result of [2] to the family given by an infinite subset of $\mathcal{D}_{-}^{1}$.

\section{ACKNOWLEDGEMENTS}

This paper was written during the author's visit to the mathematics department of the University of Liverpool. The author thanks Professor Viacheslav Nikulin for useful discussions. Thanks also go to the referee for useful suggestions.

\section{REFERENCES}

[1] A. Beauville, Variétés Kähleriennes dont la première classe de Chern est nulle, J. Differential Geom. 18(1983), no. 4, 755-782. MR.730926 (86c:32030)

[2] C.G. Madonna, On some moduli spaces of bundles on K3 surfaces, Monatsh. Math. 146(2005), 333-339. MR 2191732 (2006j:14061)

[3] C. Madonna and V.V. Nikulin, On a classical correspondence between K3 surfaces, Proc. Steklov Inst. of Math. 241(2003), 120-153. MR2024049 (2004m:14080)

[4] C.G. Madonna and V.V. Nikulin, Explicit correspondences of a K3 surface with itself, Izv. Math. 72(2008), no. 3, 497-508. MR2432754 (2009e:14061)

[5] Sh. Mukai, On the moduli space of bundles on K3 surfaces I, in: Vector bundles on algebraic varieties, Tata Inst. Fund. Res. Studies in Math. 11 (1987), 341-413. MR893604 (88i:14036)

[6] Sh. Mukai, Moduli of vector bundles on K3 surfaces and symplectic manifolds, Sugaku Expositions 1(1988), no. 2, 139-174. MR922020 (89h:32057)

[7] V.V. Nikulin, Integral symmetric bilinear forms and some of their geometric applications, Math USSR-Izv. 14(1980), no. 1, 103-167. MR.525944 (80j:10031)

[8] V.V. Nikulin, On correspondences of a K3 surface with itself. I, Proc. Steklov Inst. of Math. 246(2004), 204-226. MR2101295 (2005j:14055)

[9] V.V. Nikulin, On correspondences of a K3 surface with itself. II, Contemporary Mathematics 422, Amer. Math. Soc., Providence, RI, 2007, 121-172. MR2296436 (2008b:14061) 
[10] V.V. Nikulin, Self-correspondences of a K3 surface via moduli of sheaves, in: Y. Tschinkel and Y. Zarhin (eds.), Algebra, Arithmetic, and Geometry. Volume II: In Honor of Yu. I. Manin, 2009, Birkhäuser Boston, 439-464. MR2641198 (2011g:14032)

[11] A.N. Tyurin, Cycles, curves and vector bundles on algebraic surfaces, Duke Math. J. 54(1987), no. 1, 1-26. MR.885772 (88m:14004)

[12] K.Yoshioka, Some examples of Mukai's reflections on K3 surfaces, J. Reine Angew. Math. 515(1999), 97-123. MR1717621 (2000h:14028)

Faculty of Teacher Training and Education, Autonoma University of Madrid, Campus de Cantoblanco, C/Fco. Tomas y Valiente 3, Madrid E-28049, Spain

E-mail address: carlo.madonna@uam.es 